\title{
A UNIFIED ANALYSIS OF CONVERTERS WITH RESONANT SWITCHES
}

\author{
STEVE FREELAND AND R.D. MIDDLEBROOK
}

\author{
Power Electronics Group \\ California Institute of Technology
}

\begin{abstract}
$A B S T R A C T$
Quasi-resonant converters are a recently introduced family of single-switch resonant converters featuring zero-current or zero-voltage switching. Recognition of the topological structure uniting these converters-and the PWM converters on which they are based-leads to general models of their dc and lowfrequency ac behavior. An expression is derived that yields the $d c$ conversion ratio of a quasi-resonant converter in terms of the well-known conversion ratio of the underlying PWM topology. A small-signal, low-frequency dynamic model is developed whose parameters also incorporate the $P W M$ conversion ratio. The $d c$ and ac models reveal that any quasi-resonant converter with a full-wave resonant switch has dc and low-frequency behavior identical to that of its $P W M$ parent, with switching frequency control replacing duty ratio control. Converters with half-wave resonant switches act more like $P W M$ converters under current programming or discontinuous conduction mode, exhibiting lossless damping in the small-signal model and output resistance at dc.
\end{abstract}

\section{Introduction}

The constant demand for smaller and lighter power converters is inexorably pushing switching frequencies higher and higher. Frequencies well into the megahertz range are currently found in commercial power supplies, while experimental converters switch even faster. Such high switching frequencies are made possible by resonant topologies. In contrast to the sharp-edged, quasi-rectangular switching waveforms of PWM converters, resonant converter topologies feature smooth, quasi-sinusoidal waveforms. These smooth waveforms bring the benefits of reduced switching losses, less interference, and insensitivity to dangerous parasitics.

Resonant converters have been known and used for decades, but recent developments contain some fundamentally different features. In particular, the family of dc-dc converters with resonant switches, known as quasi-resonant converters $[3,4,5,6]$ is a departure from previous circuits $[1,2]$. The most striking feature of the new converters is the wide variety of topologies available. A recent paper, for example, illustrated no less than 26

This work was conducted under the Power Electronics Program supported by grants from GTE Communication Systems Corp., Rockwell Inc., and EG\&G Almond Instruments, Inc. different topologies [6]. While such a variety offers designers many choices, it also presents something of a problem-how to assimilate, understand, and analyze so many converter circuits.

The purpose of this paper is to recognize the common structure and features of converters with resonant switches, and to use these properties to develop general dc and small-signal models of quasi-resonant converters. An example in Section 2 will illustrate the operation and features of a quasi-resonant converter. A general approach to the analysis of these converters begins in Section 3 with a discussion of the switching process in PWM converters. Section 4 describes how a quasiresonant converter is made by creating a resonant switch in a PWM converter. The topological rules governing the resonant switch are used to derive the waveforms common to every resonant switch. In Section 5, these common waveforms lead to an equation for the dc conversion ratio of any quasi-resonant converter, an expression which exposes the important differences between half- and full-wave switches. A low-frequency smallsignal dynamic model for quasi-resonant converters is developed in Section 6. Both the dc and ac models are extended to zerovoltage-switching resonant converters in Section 7 by application of duality principles. The small-signal model is verified in Section 8 by frequency response measurements of experimental resonant converters. Section 9 summarizes the properties of quasi-resonant converters revealed by these general methods of analysis.

\section{Quasi-Resonant Converters}

The resonant switch-a combination of switches and reactances producing quasi-sinusoidal waveforms - is responsible for the unusual properties of quasi-resonant converters. As an illustration of these properties, a zero-current-switching buck converter is presented in Fig. 1, along with waveforms for the switch current $i_{S}$ and diode voltage $v_{D}$. On the assumption that the output inductor has negligibly small ripple current, the converter operates as follows.

At the beginning of each cycle of the resonant switch, the transistor $Q$ is off and the diode $D_{2}$ is on. When $Q$ is turned on, current rises linearly in the transistor, with the $d i / d t$ limited by the resonant inductor $L_{r}$. The switch current rises until $i_{S}$ meets the output inductor current $I$, at which time diode $D_{2}$ turns off and the resonant elements $L_{r}$ and $C_{r}$ form a resonant circuit. The circuit resonates until $i_{S}$ falls to zero and is in- 


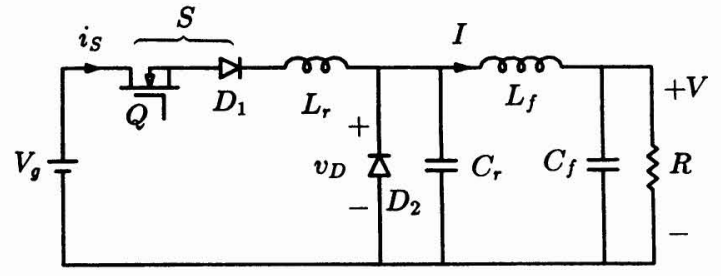

(a)
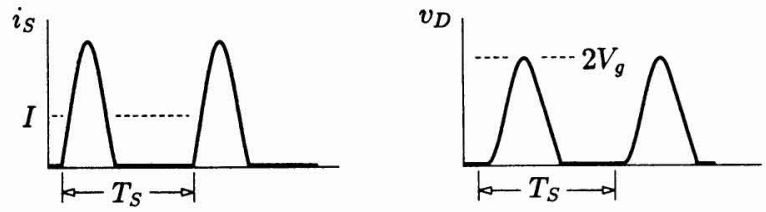

(b)

Figure 1: A quasi-resonant buck converter (a) and its switch waveforms (b).

terrupted by diode $D_{1}$. The transistor is turned off while $D_{1}$ blocks. With $Q$ and $D_{1}$ off the current $I$ discharges $C_{r}$ until the capacitor voltage falls to zero, turning on $D_{2}$. The state of the circuit is now the same as initially, and the converter idles in this condition until the control circuit turns on $Q$ to begin another cycle. The output voltage is controlled by varying the switching frequency and hence controlling how often the "pulses" of voltage in $v_{D}$ appear across the output filter formed by $L_{f}$ and $C_{f}$.

While only one of many quasi-resonant topologies, the converter of Fig. 1 illustrates the important features of a resonant switch and its effects in a power converter. First, the circuit of Fig. 1(a) is obviously a derivative of the common PWM buck topology shown in Fig. 2, differing only by the presence of the three components $D_{1}, L_{r}$, and $C_{r}$. The visual similarity is no coincidence: quasi-resonant converters are all derived by addition of these three components to a PWM converter topology.

The waveforms of Fig. 1(b) demonstrate a second feature of the resonant switch: the transistor switch turns both on and off into zero current, allowing the converter to operate at very high frequencies without excessive switching losses. Just as important as low-loss switching is the fact that the waveforms of Fig. 1(b) have finite slopes. Such waveforms may cause less interference problems than the waveforms of (ideally) infinite slope found in PWM converters. Another consequence of the continuous waveforms of Fig. 1(b) is that parasitic inductances and capacitances can occur at several places in the converter without qualitatively affecting the waveforms. In some topologies, in fact, unavoidable parasitics (such as transformer leakage inductance) can be used as part or whole of the resonant

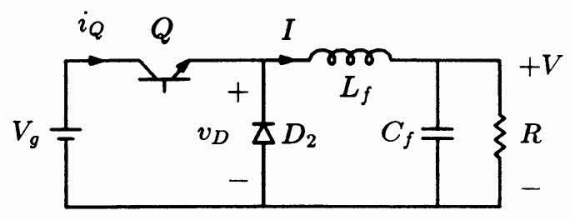

Figure 2: A PWM buck converter. reactances $L_{r}$ or $C_{r}$. This is a major advantage over PWM converters, in which parasitics can produce dangerous stresses and preclude very high switching frequencies.

A third feature of the resonant switch is that the current in $L_{r}$ and voltage on $C_{r}$ do not resonate continuously. Instead, they assume fixed values at the end of every cycle. This constraint considerably simplifies the analysis and control of quasiresonant converters compared to converters that "ring" continuously.

Converters with resonant switches must be controlled by varying the switching frequency. This is apparent from the waveforms of Fig. 1(b), which show that while the turn-on of transistor $Q$ is a matter of choice, the turn-off of $D_{1}$ is determined solely by the resonant current waveform and cannot be directly controlled.

The resonant buck converter of Fig. 1 is only one of many possible quasi-resonant converters. Any PWM topology-boost or flyback, for example-may be used instead of the buck as the PWM topology underlying the resonant converter. The twoquadrant switch consisting of $Q$ and $D_{1}$ may be "half-wave" or "full-wave" [4]. Duality can be applied to the converter to yield a circuit which has its transistor switching at zero voltage instead of zero current [5]. Finally, in any particular topology, the resonant reactances $L_{r}$ and $C_{r}$ can be placed in several different positions while preserving the benefits of resonant switching [6].

\section{PWM Switching}

The term "PWM" (pulse-width modulation) is used here not to indicate any particular control scheme, but rather as a means of distinguishing converters with quasi-rectangular waveforms. For the present purposes, a PWM converter is defined as a dcdc converter with one transistor, one diode, no losses, and small ripple in every reactance and the load, $R$. All reactances are linear. No magnetic coupling is allowed, but this restriction and the limitation to a single switch can be removed for some PWM converters-and for resonant converters derived from them-by fairly simple modifications to the analysis that follows.

From this definition of a PWM converter, one may prove the following two results [7].

Theorem 1 The transistor $Q$, the diode $D_{2}$, and a set of capacitors with total voltage $V_{\text {off }}$ form a loop. The source $V_{g}$ may also be included in the loop, with its voltage included in $V_{\text {off }}$.

Theorem 2 The transistor $Q$, the diode $D_{2}$, and a set of inductors with total current $I_{\mathrm{on}}$ form a cut-set.

The quantities $V_{\text {off }}$ and $I_{\text {on }}$ are easy to find in any particular topology: they are simply the voltage and current stresses on the transistor and diode. For example, in the buck converter of Fig. 2, $V_{\text {off }}$ is the source voltage $V_{g}$, while $I_{\text {on }}$ is the current $I$ in the output filter inductor.

Application of Kirchhoff's laws to the loop and cut-set of Theorems 1 and 2 gives

$$
\begin{aligned}
v_{Q}+v_{D} & =V_{\text {off }} \\
i_{Q}+i_{D} & =I_{\text {on }}
\end{aligned}
$$

For efficient power conversion, the transistor and diode must operate in the switched mode, i.e., each device must be either on, with zero voltage (ideally), or off, with zero current. This 

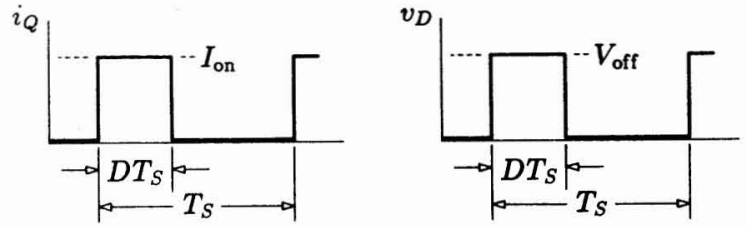

Figure 3: The transistor current and diode voltage waveforms in any $P W M$ converter.

fact, together with Eqs.(1) and (2), implies that either the transistor or diode, but not both, must be on at any given time. The duty ratio $D$ is defined as the fraction of a switching period in which the transistor is on. The waveforms for the transistor current and diode voltage in any PWM converter will be those of Fig. 3, to the extent that ripple can be neglected.

\section{Resonant Switches}

A quasi-resonant converter is the result of implementing a resonant switch in a PWM converter. The process involves adding three components to the PWM converter, according to certain topological rules. First, a diode $D_{1}$ is added either in series or in parallel with the transistor $Q$, forming a two-quadrant switch $S$. The resonant inductance $L_{r}$ and capacitance $C_{r}$ are then added.

The desired quasi-sinusoidal waveforms will appear only if $L_{r}$ and $C_{r}$ are inserted at certain locations in the PWM topology. A recent paper gave three rules governing the many possible locations [6]. Only two rules are necessary, however. The following two generate all zero-current-switching quasi-resonant topologies, i.e., those in which the transistor turns both on and off into zero current. (Zero-voltage-switching topologies are discussed in Section 7).

Rule 1 The resonant capacitor $C_{r}$ must form a loop with the diode $D_{2}$ and a (possibly empty) set of "stiff" capacitors and voltage sources.

Rule 2 The resonant inductor $L_{r}$ must form a cut-set with the two quadrant switch $S$ and a (possibly empty) set of "stiff" inductors.

A "stiff" capacitor or inductor is one with small switching ripple. All non-resonant capacitors and inductors are assumed stiff, as in the original PWM converter. As pointed out in [6], Rule 1 ensures that the diode voltage will be continuous. Rule 2 (not found in [6]) similarly imposes continuity on the transistor current.

Two further results may be derived from Rules 1 and 2, relating the topology of the resonant switch to that of the original PWM converter [7]:

Theorem 3 The elements $S, D_{2}, L_{r}$, and the elements producing the voltage $V_{\text {off }}$ together form a loop.

Theorem 4 The elements $S, D_{2}, C_{r}$, and the inductors producing $I_{\text {on }}$ together form a cut-set.

These two results imply that $L_{r}$ is inserted in series with elements of the PWM converter, while $C_{r}$ is added in parallel with the PWM elements to form a resonant switch. Theorems 3 and 4 hold for both zero-current and zero-voltage-switching converters.

Figure 1(a) verifies that these topological restrictions are satisfied for the resonant buck converter. The loop of Rule 1 contains only $C_{r}$ and $D_{2}$ and the cut-set of Rule 2 contains only $L_{r}$ and $S$. The loop of Theorem 3 contains $S, D_{2}, L_{r}$, and the source $V_{g}$ as $V_{\text {off }}$. The cut-set of Theorem 4 contains $S, D_{2}, C_{r}$, and the filter inductor which carries the current $I_{\mathrm{on}}$.

Define $i_{S}, i_{D}, i_{C}$, and $i_{L}$ as the currents in the switch $S$, diode $D_{2}$, capacitor $C_{r}$, and inductor $L_{r}$, respectively. Similarly define $v_{S}, v_{D}, v_{C}$, and $v_{L}$ as the voltages across these elements. Application of Kirchhoff's laws to Rules 1 and 2 and to Theorems 3 and 4 yields the following equations describing a resonant switch.

$$
\begin{aligned}
v_{D}-v_{C} & =V_{\mathrm{dc}} \\
i_{S}-i_{L} & =I_{\mathrm{dc}} \\
v_{S}+v_{D}+v_{L} & =V_{\mathrm{off}} \\
i_{S}+i_{D}-i_{C} & =I_{\mathrm{on}}
\end{aligned}
$$

where the small-ripple quantities $V_{\mathrm{dc}}$ and $I_{\mathrm{dc}}$ are generated by the (possibly empty) sets of "stiff" elements in Theorems 3 and 4. Both these sets are empty for the example of Fig. 1, so that $V_{\mathrm{dc}}$ and $I_{\mathrm{dc}}$ are both zero for this converter.

Although $V_{\mathrm{dc}}$ and $I_{\mathrm{dc}}$ will depend upon the location of $L_{r}$ and $C_{r}$ within a given topology, the actual values are of no concern here. What is important is that the quantities on the right-hand sides of Eqs.(3) through (6) are all dc, given the assumption of small ripple in all reactances but $L_{r}$ and $C_{r}$.

These four equations are sufficient to derive the waveforms $i_{S}(t)$ and $v_{D}(t)$ for any zero-current resonant switch. To avoid losses, either $i_{D}$ or $v_{D}$ must be zero at any time, depending on whether the diode is on or off. Likewise, either $i_{S}$ or $v_{S}$ must be zero. Application of these dichotomies to Eqs.(3) through (6) produces the four states of the resonant switch shown in Table 1 . The equations describing the waveforms $i_{S}$ and $v_{D}$ during each interval are given in Table 2, which uses the following definitions:

$$
\begin{aligned}
\omega_{0} & \equiv \frac{1}{\sqrt{L_{r} C_{r}}} \\
R_{0} & \equiv \sqrt{L_{r} / C_{r}} \\
\rho & \equiv \frac{R_{0} I_{\text {on }}}{V_{\text {off }}} .
\end{aligned}
$$

The frequency $F_{0}=\omega_{0} / 2 \pi$ is the resonant frequency and $R_{0}$ the characteristic resistance of the resonant tank formed by $L_{r}$ and $C_{r}$ during the resonant interval. The operating point of the resonant switch is indicated by the dimensionless quantity $\rho$, a

\begin{tabular}{|r|c|c|c|}
\hline & & \multicolumn{2}{|c|}{ Switch State } \\
\cline { 3 - 4 } Interval & Name & $S$ & $D_{2}$ \\
\hline Initial State & Idle & OFF & ON \\
$0<t<T_{1}$ & Charging & ON & ON \\
$T_{1}<t<T_{2}$ & Resonant & ON & OFF \\
$T_{2}<t<T_{3}$ & Discharging & OFF & OFF \\
$T_{3}<t<T_{S}$ & Idle & OFF & ON \\
\hline
\end{tabular}

Table 1: The resonant switch takes on four different states during each switching period. 


\begin{tabular}{|c|c|c|c|}
\hline Interval & $\begin{array}{c}\text { Switch } \\
\text { Current } \\
i s(t) / I_{\text {on }}\end{array}$ & $\begin{array}{c}\text { Diode } \\
\text { Voltage } \\
v_{D}(t) / V_{\text {off }}\end{array}$ & $\begin{array}{c}\text { Duration } \\
\omega_{0} \Delta t\end{array}$ \\
\hline \hline Charging & $\frac{1}{\rho} \theta$ & 0 & $\rho$ \\
\hline Resonant & $1+\frac{1}{\rho} \sin \theta$ & $1-\cos \theta$ & $\left\{\begin{array}{c}\pi+\sin ^{-1} \rho \\
\text { half-wave) } \\
2 \pi-\sin ^{-1} \rho \\
(\text { full-wave) }\end{array}\right.$ \\
\hline $\begin{array}{c}\text { Discharging } \\
\text { half-wave: } \\
\text { full-wave: }\end{array}$ & 0 & $1+\sqrt{1-\rho^{2}}-\rho \theta$ & $\frac{1}{\rho}\left(1+\sqrt{1-\rho^{2}}\right)$ \\
\hline Idle & 0 & $1-\sqrt{1-\rho^{2}}-\rho \theta$ & $\frac{1}{\rho}\left(1-\sqrt{1-\rho^{2}}\right)$ \\
\hline
\end{tabular}

Table 2: Summary of the equations describing the behavior of the zero-current resonant switch. The quantity $\theta$ is the product of $\omega_{0}$ and the time elapsed since the beginning of an interval.

factor somewhat akin to the quality factor of a series resonant circuit.

The waveforms $i_{S}$ and $v_{D}$ are shown in Fig. 4 for half-wave and full-wave switches. The half-wave switch interrupts the current $i_{S}$ when it first reaches zero, whereas the full-wave switch allows the current $i_{S}$ to go negative. In the full-wave switch, the transistor is turned off while $D_{1}$ is conducting, so that the current $i_{S}$ is stopped when it reaches zero from below. If $\rho>1$ for either kind of switch, the current $i_{S}$ never returns to zero and the switch $S$ cannot turn off into zero current. Operation of the resonant switch therefore requires that $\rho \leq 1$. For the mode of operation shown in Fig. 4 and assumed throughout this paper, the voltage $v_{D}$. must reset to zero before $Q$ is turned on to begin the next cycle. This restriction limits the maximum switching frequency of the resonant switch.

It is important to note that the equations of Table 2 are independent of $V_{\mathrm{dc}}$ and $I_{\mathrm{dc}}$ of Eqs.(3) and (4). The waveforms $i_{S}$

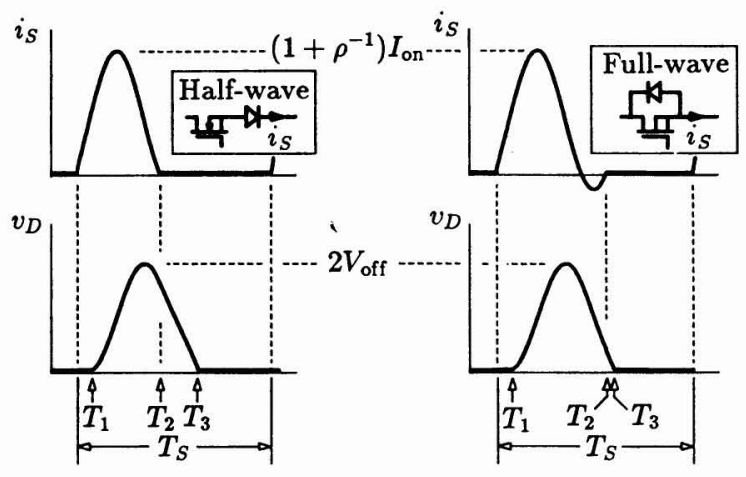

(a)

(b)

Figure 4: Switching waveforms in a quasi-resonant converter: half-wave (a), and full-wave (b). The upper curves show the current in the two-quadrant switch $S$ (shown in the insets), and the lower curves are the voltage across the diode $D_{2}$.

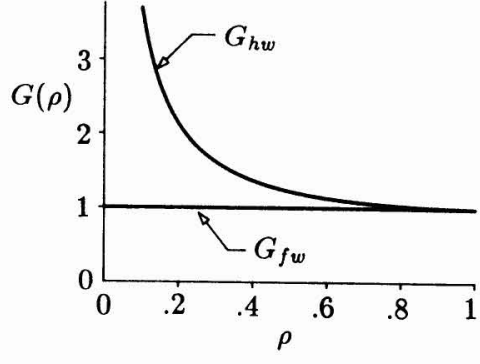

Figure 5: Plots of the functions $G_{h w}$ and $G_{f w}$ of Eqs.(12) and (19).

and $v_{D}$ are therefore independent of the location of the resonant reactances within a topology.

While the equations of Table 2 are included for completeness, the only quantities needed in the following analysis are the averages of $i_{S}$ and $v_{D}$ over a single cycle. These are given by

$$
\begin{aligned}
\bar{\imath}_{S} & =I_{\text {on }} \frac{F_{S}}{F_{0}} G(\rho) \\
\bar{v}_{D} & =V_{\text {off }} \frac{F_{S}}{F_{0}} G(\rho)
\end{aligned}
$$

where $F_{S}=1 / T_{S}$ is the switching frequency, and the function $G$ depends on the type of switch $S$. If $G_{h w}$ and $G_{f w}$ denote the functions appropriate for the half-wave and full-wave switches, respectively, these functions are given by

$$
\begin{array}{r}
G_{h w}(\rho)=\frac{1}{2 \pi}\left[\frac{\rho}{2}+\pi+\sin ^{-1} \rho+\frac{1}{\rho}\left(1+\sqrt{1-\rho^{2}}\right)\right] \\
G_{f w}(\rho)=\frac{1}{2 \pi}\left[\frac{\rho}{2}+2 \pi-\sin ^{-1} \rho+\frac{1}{\rho}\left(1-\sqrt{1-\rho^{2}}\right)\right]
\end{array}
$$

The two functions are plotted in Fig. 5, revealing a striking difference between the half-wave and full-wave switches. The function $G_{f w}$ is virtually independent of $\rho$, so that the averages ${ }^{{ }^{i}} S / I_{\text {on }}$ and $\bar{v}_{D} / V_{\text {off }}$ are functions of frequency alone, independent of the converter load. In contrast, $G_{h w}$ depends heavily on $\rho$. The large difference in $G_{h w}$ and $G_{f w}$ is somewhat surprising given the similarities of the waveforms in Fig. 4, but the difference is real and has important consequences in the dc and small-signal ac behavior of quasi-resonant converters.

A resonant switch is not limited to a single inductance $L_{r}$ or capacitance $C_{r}$. So long as each resonant reactance individually satisfies Rules 1 or 2 , any number of resonant inductors and capacitors can be added to a PWM topology, resulting in a quasi-resonant converter with effective values of $L_{r}$ and $C_{r}$ equal to the sum of all the resonant inductances and capacitances, respectively. Quasi-resonant topologies are therefore insensitive to any parasitic reactances that appear in positions satisfying Rules 1 or 2 . The only effect of such parasitics is to modify the values of $L_{r}$ and $C_{r}$. The waveforms of the converter are qualitatively unchanged, and all the advantages of resonant switching remain in effect.

\section{Dc Analysis}

The average behavior of the resonant switch is used in this section to find the dc conversion ratio of any zero-current quasi- 


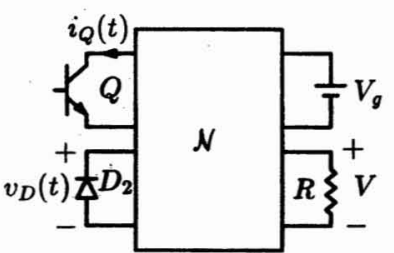

(a)

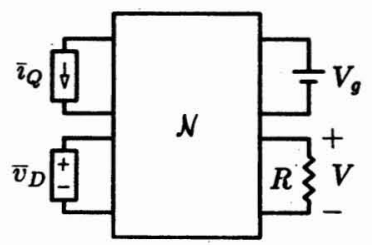

(b)

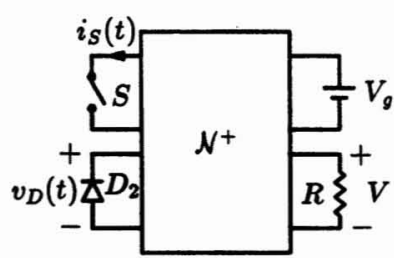

(c)

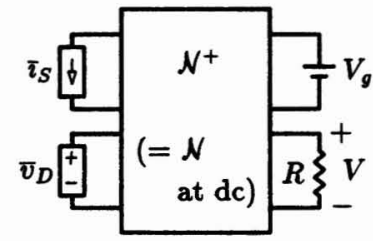

(d)
Figure 6: A PWM converter (a) and a dc equivalent circuit (b); a quasi-resonant converter (c) and a dc equivalent circuit (d).

resonant converter in terms of the conversion ratio of its PWM parent.

\subsection{The De Conversion Ratio}

Consider the diagram of Fig. 6(a) which views a PWM converter as a linear, time-invariant network $\mathcal{N}$ connected to the timevarying devices $Q$ and $D_{2}$. The network $\mathcal{N}$ has three inputs$V_{g}$, the current $i_{Q}(t)$ and the voltage $v_{D}(t)$-and a single output $V$, the voltage across the load. The dc conversion ratio of the PWM converter is defined as $M \equiv V / V_{g}$.

Since the network $\mathcal{N}$ is linear, the average of its output can be found by driving the network with only the averages of its inputs. Specifically, the conversion ratio $M$ is unchanged if $Q$ and $D_{2}$ are replaced with sources generating the averages of $i_{Q}$ and $v_{D}$, as shown in Fig. 6(b). These average values are easily found from the waveforms of Fig. 3:

$$
\begin{aligned}
\bar{\imath}_{Q} & =D I_{\text {on }} \\
\bar{v}_{D} & =D V_{\text {off }} .
\end{aligned}
$$

The conversion ratio $M$ of a PWM converter is known to be a function only of the duty ratio $D$. Let $M_{P}(D)$ denote this function so that

$$
M=M_{P}(D)
$$

for a PWM converter. The functions $M_{P}$ are well-known for common PWM topologies. For example, $M_{P}(D)$ is $D$ for a buck topology, $1 /(1-D)$ for the boost, and $D /(1-D)$ for the buck-boost and Cuk topologies.

Figure 6(c) shows a quasi-resonant converter generated from the PWM converter of Fig. 6(a). The linear network $\mathcal{N}^{+}$of the quasi-resonant converter is the same as the PWM network $\mathcal{N}$ with the exception of the addition of $L_{r}$ in series and $C_{r}$ in parallel with the elements of $\mathcal{N}$. At dc, however, the networks $\mathcal{N}$ and $\mathcal{N}^{+}$are equivalent, because $L_{r}$ supports no average voltage and $C_{r}$ carries no average current.

The conversion ratio of the resonant converter is unchanged by replacing the switch $S$ and diode $D_{2}$ with sources producing the averages $\bar{i}_{S}$ and $\bar{v}_{D}$, as shown in Fig. $6(\mathrm{~d})$. The averages $\bar{i}_{S}$ and $\bar{v}_{D}$ are given by Eqs.(10) and (11).
Since there is no difference at dc between Figs. 6(b) and 6(d), a quasi-resonant converter and its PWM parent must have the same conversion ratio $M$ whenever the averages $\bar{i}_{S}$ (or $\bar{i}_{Q}$ ) and $\bar{v}_{D}$ are the same in the two converters. Equations (10), (11), (14), and (15) reveal that this is so whenever

$$
D=\frac{F_{S}}{F_{0}} G(\rho)
$$

Use of Eq.(17) to replace $D$ in Eq.(16) gives an expression for the conversion ratio of the resonant converter,

$$
M=M_{P}\left(\frac{F_{S}}{F_{0}} G(\rho)\right) .
$$

This equation is not immediately useful, however, because $\rho$ involves the ratio of $I_{\text {on }}$ and $V_{\text {off }}$, which in turn depends on the conversion ratio. A relation between $\rho$ and $M$ will solve this problem. For every PWM converter, as defined in Section 3, (see, for example, [10] for a catalog of many such converters), the following is true:

$$
\frac{V_{\text {off }}}{I_{\text {on }}}=\frac{R}{M},
$$

where $R$ is the ratio of dc load voltage and current. Use of this expression in Eq.(9), the definition of $\rho$, gives

$$
\rho=\frac{R_{0} I_{\text {on }}}{V_{\text {off }}}=M \frac{R_{0}}{R} .
$$

Substitution of Eq.(20) in Eq.(18) and solution for $F_{S} / F_{0}$ yields

$$
\frac{F_{S}}{F_{0}}=\frac{D_{P}(M)}{G\left(M R_{0} / R\right)},
$$

where $D_{P}(M)$ is the inverse of the PWM conversion-ratio function $M_{P}(D)$. For example, in the buck-boost converter, the conversion ratio is $M_{P}(D)=D /(1-D)$, and the inverse relation is $D_{P}(M)=M /(1+M)$. For a buck converter $M_{P}(D)=D$, so that $D_{P}(M)$ is simply $M$.

Equation (21) enables one to find the conversion ratio of any zero-current-switching resonant-switch converter knowing only the quantities $R, R_{0}$, and the well-known PWM conversion function $M_{P}(D)$. (Section 7 shows how Eq.(21) is modified for zero-voltage converters.) The presence of the PWM function $D_{P}(M)$ in Eq.(21) highlights the fact that a PWM topology underlies each resonant-switch converter.

For the quasi-resonant buck converter of Fig. 1, the function $D_{P}(M)$ is simply $M$ and the conversion ratio is governed by $F_{S} / F_{0}=M / G\left(\frac{M R_{0}}{R}\right)$. The conversion ratio of this converter is plotted in Fig. 7.

\subsection{Implications of the Conversion Ratio}

Half-wave and full-wave switches produce very different dc behavior, as the example of Fig. 7 shows. For a converter with a full-wave switch, the function $G_{f w}$ is nearly unity for any permissible value of its argument, as shown in Fig. 5. With $G_{f w}=1$, Eq.(18) becomes

$$
M=M_{P}\left(F_{S} / F_{0}\right) .
$$

The conversion ratio of the resonant converter is found simply by replacing $D$ with $F_{S} / F_{0}$ in the function $M_{P}$.

The conversion ratio of a full-wave converter will exhibit the same features as that of the PWM parent, including voltage step-up or step-down capabilities, linearity or nonlinearity 


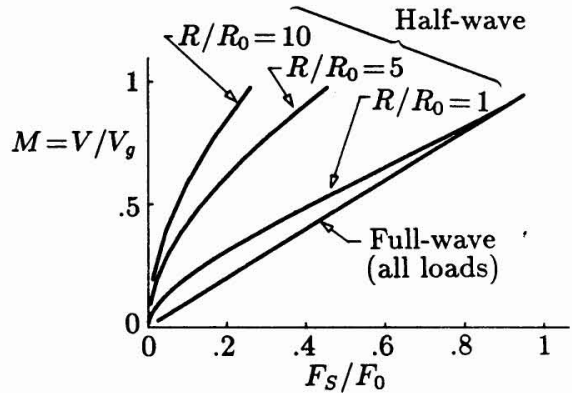

Figure 7: Dc conversion ratio of the quasi-resonant buck converter.

with respect to the control input, and a lack of load dependence. The curves of Fig. 7, for example, show that the full-wave buck converter has a conversion ratio less than 1 , proportional to control $\left(F_{S} / F_{0}\right)$, and independent of load, just like the PWM buck converter. Although insertion of a full-wave resonant switch in a PWM converter radically changes the converter's "internal" waveforms, the only "external" effect at dc is the replacement of the PWM control parameter $D$ with the resonant control parameter $F_{S} / F_{0}$. The same effect will be seen in half-wave converters as $\rho \rightarrow 1$, since $G_{h w}$ and $G_{f w}$ converge in that limit.

Half-wave resonant-switches have very different properties since the function $G_{h w}$ depends heavily on $\rho$, as seen in Fig. 5 . For example, the curves of Fig. 7 show that the conversion ratio of a half-wave buck converter is neither linear in control nor independent of load. A converter with a load-dependent conversion ratio has an output resistance, defined by the variation of output voltage with changing load current while the control input is held constant. Application of the definition of output resistance to Eq.(21) yields

$$
R_{\mathrm{out}}=\left[\frac{m D_{P}(M)}{M D_{P}^{\prime}(M)}\right] R,
$$

where $D_{P}^{\prime}$ is the derivative of $D_{P}$ with respect to the argument $M$ (note that $D^{\prime}$ is not $1-D$ ), and $m$ is defined by

$$
m \equiv \rho \frac{\left|G^{\prime}(\rho)\right|}{G(\rho)} .
$$

For the half-wave switch, the derivative of $G$ is

$$
G_{h w}^{\prime}(\rho)=-\frac{1}{4 \pi}\left(\frac{1+\sqrt{1-\rho^{2}}}{\rho}\right)^{2} .
$$

Figure 8 shows $m$ for a half-wave resonant switch. For a fullwave resonant switch, $G_{f w}$ is constant so that $G^{\prime}, m$, and the output resistance are all zero.

For the half-wave buck converter of Fig. $1, D_{P}(M)$ is simply $M$, and the output resistance is $m$ times the dc load resistance $R$. At low values of $\rho$, the output resistance is substantial.

The output resistance introduced by a half-wave resonant switch is a "lossless" resistance, much like that produced by current programming and discontinuous conduction mode in PWM converters. The effect can be used to advantage when several converters are operated in parallel. The output resistances of half-wave resonant-switch converters will promote currentsharing when several converters are tied to the same load and operated with the same switching frequency.

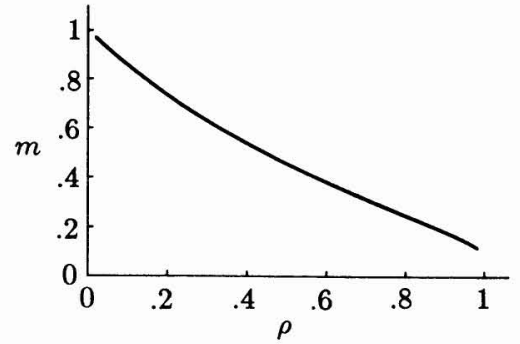

Figure 8: Plot of $m$ as a function of $\rho$. The quantity $m$ appears in expressions for the output resistance and in the small-signal parameters of Section 6.

\section{Ac Analysis}

The waveforms of the resonant switch violate the assumptions of most averaged ac models of PWM converters (such as statespace averaging [8]). The goal of this section is to develop a small-signal ac model of quasi-resonant converters that is valid for frequencies well below the switching frequency.

\subsection{Equivalent Waveforms}

With reference again to Fig. 6(c), a quasi-resonant converter can be viewed as the linear network $\mathcal{N}^{+}$driven by the sources $i_{S}(t)$ and $v_{D}(t)$. When the resonant converter is modulated, $I_{\text {on }}$ and $V_{\text {off }}$ become the time-varying quantities $i_{\text {on }}(t)$ and $v_{\text {off }}(t)$. Modulation is assumed to occur only at frequencies well below the switching frequency, however, so that $i_{\text {on }}(t)$ and $v_{\text {off }}(t)$, as well as all the non-resonant states of the network $\mathcal{N}^{+}$, vary slowly. (These quantities are already assumed to have small switching ripple.)

Only the low-frequency behavior of the network outputs are of interest, and hence only the low-frequency components of the inputs $i_{S}(t)$ and $v_{D}(t)$ are needed. Any two waveforms with the same low-frequency spectra as $i_{S}(t)$ and $v_{D}(t)$ can be applied to the ports of the network $\mathcal{N}^{+}$without changing the low-frequency behavior of the network states and outputs.

It is postulated that the "averaged" waveforms

$$
\begin{aligned}
\bar{\imath}_{S}(t) & =i_{\text {on }}(t) \frac{f_{S}(t)}{F_{0}} G\left[R_{0} i_{\text {on }}(t) / v_{\text {off }}(t)\right] \\
\bar{v}_{D}(t) & =v_{\text {off }}(t) \frac{f_{S}(t)}{F_{0}} G\left[R_{0} i_{\text {on }}(t) / v_{\text {off }}(t)\right]
\end{aligned}
$$

contain the same low-frequency information as the actual switching waveforms $i_{S}(t)$ and $v_{D}(t)$, where $f_{S}(t)$ is a continuous, low-frequency approximation of the switching frequency. This assumption can be justified using the approximations of small switching ripple and low-frequency modulation, provided $v_{D}(t)$ and $i_{S}(t)$ are sufficiently band-limited [7].

\subsection{Small-Signal Model}

The small-signal behavior of a quasi-resonant converter is found by linearizing Eqs.(26) and (27), the equivalent low-frequency waveforms replacing $i_{S}(t)$ and $v_{D}(t)$. Each equation is expanded as a function of the quantities $\hat{\imath}_{\text {on }}, \hat{v}_{\text {off }}$, and $\hat{f}_{S}$, where a hat $\left({ }^{\wedge}\right)$ signifies the small-signal variation of a quantity. For 
example, the variation of the switch current, $\hat{\bar{i}}_{S}$, is given by

$$
\hat{\bar{i}}_{S}=\frac{\partial \bar{i}_{S}}{\partial i_{\text {on }}} \hat{i}_{\text {on }}+\frac{\partial \bar{i}_{S}}{\partial v_{\text {off }}} \hat{v}_{\text {off }}+\frac{\partial \bar{i}_{S}}{\partial f_{S}} \hat{f}_{S}
$$

with a similar expansion for $\hat{\bar{v}}_{D}$. Expressions for the partial derivatives can be simplified considerably by use of Eq.(21) from Section 5 to eliminate the ratio $F_{S} / F_{0}$. The parameter $m$, defined in Section 5 and plotted in Fig. 8, also can be used to advantage. Evaluation of the partial derivatives yields

$$
\begin{aligned}
h_{i i} & \equiv \frac{\partial \bar{i}_{S}}{\partial I_{\text {on }}}=(1-m) D_{P}(M) \\
h_{i v} & \equiv \frac{\partial \bar{\imath}_{S}}{\partial V_{\text {off }}}=\frac{m M D_{P}(M)}{R} \\
h_{i f} & \equiv \frac{\partial \bar{i}_{S}}{\partial F_{S}}=\frac{I_{\text {on }} D_{P}(M)}{F_{S}} \\
h_{v i} & \equiv \frac{\partial \bar{v}_{D}}{\partial I_{\text {on }}}=-\frac{m R D_{P}(M)}{M} \\
h_{v v} & \equiv \frac{\partial \bar{v}_{D}}{\partial V_{\text {off }}}=(1+m) D_{P}(M) \\
h_{v f} & \equiv \frac{\partial \bar{v}_{D}}{\partial F_{S}}=\frac{V_{\text {off }} D_{P}(M)}{F_{S}}
\end{aligned}
$$

The units of each $h$-parameter can be found by imagining a fraction bar $(/)$ between the two subscripts. For instance, $h_{v i}$ has units of $v / i$, or resistance. The function $D_{P}(M)$ is the inverse of the PWM conversion-ratio function, as described in Section 5 .

A small-signal circuit model for a quasi-resonant converter is shown in Fig. 9. Each term of Eq.(28) is represented by a controlled current source of value given by the appropriate $h$-parameter. These three sources replace the switch $S$, while three voltage sources replace the diode $D_{2}$. Frequency variation $\hat{f}_{S}$ is considered an independent parameter, and the sources it controls are indicated by circles. Sources dependent upon voltages and currents elsewhere in the circuit are indicated by rectangles.

\subsection{Interpretation of the Small-Signal Model}

The small-signal model of Fig. 9 has several important features that are not readily apparent. As a means of exposing these

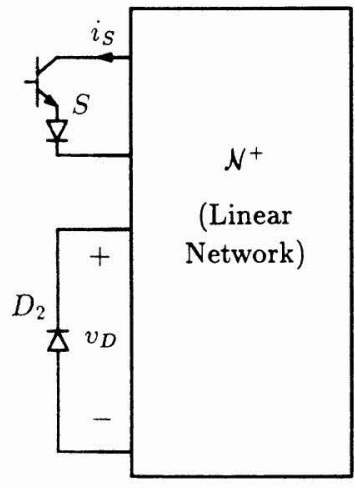

(a)

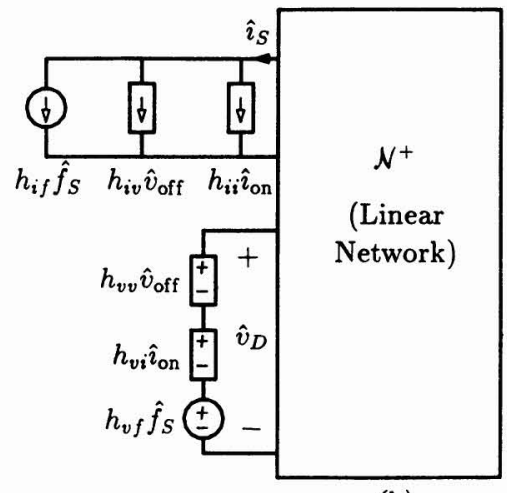

(b)
Figure 9: The small-signal model of a quasi-resonant converter is found by replacing the switch $S$ and diode $\mathrm{D}_{2}$ of the converter circuit (a) with controlled sources as in (b).

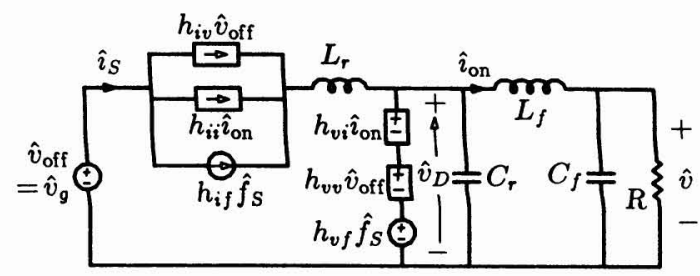

(a)

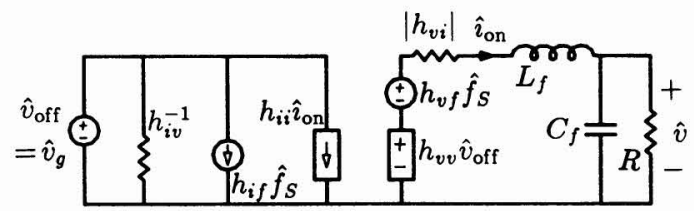

(b)

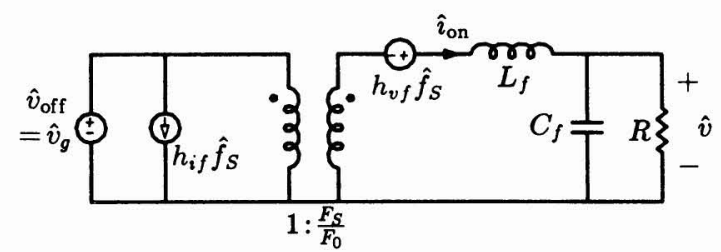

(c)

Figure 10: Small-signal circuit model of the quasi-resonant buck converter (a). Manipulation of the controlled sources leads to the equivalent circuit of (b). For a full-wave converter, the model further reduces to the circuit of (c).

features, Fig. 10 shows the small-signal circuit model of the resonant buck converter of Fig. 1(a). In Fig. 10(a), all voltages and currents have been replaced by their small-signal variations. Controlled sources take the place of the two-quadrant switch $S$ and diode $D_{2}$ according to Fig. 9 .

In Fig. 10(a), the resonant capacitor $C_{r}$ appears across three ideal voltage sources, while $L_{r}$ is in series with ideal current sources. Consequently, neither $C_{r}$ nor $L_{r}$ has any effect on the model and these elements may be removed. In particular, note that $C_{r}$ and $L_{r}$ do not increase the order of the small-signal model; they do not contribute state variables to the system.

This result is general. Rule 1 of Section 4 implies that, in any zero-current-switching quasi-resonant converter, $C_{r}$ is in a loop of capacitors and voltage sources in the small-signal model. The voltage on $C_{r}$ is therefore dependent on the states and inputs of the circuit model and cannot be a state variable. Rule 2 likewise puts $L_{r}$ in a cut-set of inductors and current sources, making the current in $L_{r}$ dependent. The order of the small-signal model for a quasi-resonant converter will therefore be the same as that of the state-space-averaged model [8] (or other low-frequency model) of the parent PWM converter.

Even though $L_{r}$ and $C_{r}$ do not increase the system order, under some conditions these elements can still appear in the small-signal circuit model. For instance, another possible location (satisfying Rule 1) for $C_{r}$ in the buck topology is across the filter inductor $L_{f}$. In this position, $C_{r}$ and $L_{f}$ form an undamped parallel resonant circuit, inserting a "notch" in the converter's transfer functions. If $L_{f}$ is large enough, the "notch" can appear at frequencies well below the switching frequency. 
Even though the order of the circuit is not increased by the presence of $C_{r}$, the resonant capacitor can nevertheless have a significant effect on the response.

This somewhat unusual example demonstrates that one cannot summarily remove $C_{r}$ and $L_{r}$ from the small-signal circuit model. In most cases, however, $L_{r}$ and $C_{r}$ will have negligible effects on the response, either because of their topological position or because their values are so small that they have no effect at frequencies where the model is valid.

Removal of $L_{r}$ and $C_{r}$ from Fig. 10(a) and manipulation of the controlled sources leads to the equivalent circuit shown in Fig. 10(b). When the converter has a half-wave switch, as shown in Fig. 1(a), the resistances $h_{i v}^{-1}$ and $\left|h_{v i}\right|$ of Fig. 10(b) are both positive, and the sources containing $h_{i i}$ and $h_{v v}$ cannot be combined into a transformer. The network is lossy and non-reciprocal. The presence of the resistors demonstrates an important result: half-wave converters have lossless damping of their transfer functions. The effect is much like that found in PWM converters with discontinuous conduction mode [9], current programming [12], or storage-time modulation [13]. No power is dissipated in the switches or reactances of the converter, but modulation of the switching instants tends to remove perturbations faster than in the "undamped" converter.

As $\rho \rightarrow 1$, the half-wave resonant-switch waveforms approach those of a full-wave converter. Both $h_{i v}$ and $h_{v i}$ approach zero and the damping disappears. If the converter is outfitted with a full-wave switch ( $D_{1}$ anti-parallel to the transistor), $m=0$ so that $h_{v v}$ and $h_{i i}$ are both equal to $D_{P}(M)$, which in turn equals $F_{S} / F_{0}$ for the full-wave converter. The sources containing $h_{v v}$ and $h_{i i}$ constitute an ideal transformer, as shown in the equivalent circuit of Fig. 10(c). With the equivalence of $f_{S} / F_{0}$ and $d$ as control variables, the model of Fig. 10(c) agrees with the state-space-averaged model of the PWM buck converter [8].

This again is a general result. Full-wave converters, so long as $L_{r}$ and $C_{r}$ do not affect the response, have the same small-signal dynamics as their PWM parent converters. When $f_{S} / F_{0}$ and $d$ are equated, the small-signal model suggested in Fig. 9 agrees with "averaged" models of the parent PWM converter $[10,11]$. To the extent that the small-ripple approximation is met, the state-space averaged model [8] is also equivalent.

\section{Zero-Voltage Resonant Switches}

The dual of a zero-current-switching resonant switch is a zerovoltage switch, a circuit that allows the switching transistor to turn both on and off into zero voltage. Zero-voltage switches can be studied by applying duality to the development of the zero-current switch, including the dc and ac models. The duality transformation imposes the changes listed in Table 3 . Under these changes, Rules 1 and 2 become the following two rules defining allowable locations of the resonant reactances in a zerovoltage resonant converter:

Rule 3 The resonant inductor $L_{r}$ must form a cut-set with the diode $\mathrm{D}_{2}$ and a (possibly empty) set of "stiff" inductors.

Rule 4 The resonant capacitor $C_{r}$ must form a loop with the two-quadrant switch $S$ and a (possibly empty) set of "stiff" capacitors and voltage sources.

Theorems 3 and 4 are unchanged by duality. As with the zerocurrent converters, the reactances $L_{r}$ and $C_{r}$ have several possible positions within a given topology.

\begin{tabular}{|ccc|}
\hline Zero-Current & & Zero-Voltage \\
\hline$I_{\text {on }}$ & $\longrightarrow$ & $V_{\text {off }}$ \\
$V_{\text {off }}$ & $\longrightarrow$ & $I_{\text {on }}$ \\
$R_{0}$ & $\longrightarrow$ & $R_{0}^{-1}$ \\
$R$ & $\longrightarrow$ & $R^{-1}$ \\
$\rho$ & $\longrightarrow$ & $\rho^{-1}$ \\
$i_{S}$ & $\longrightarrow$ & $v_{S}$ \\
$v_{D}$ & $\longrightarrow$ & $i_{D}$ \\
$M$ & $\longrightarrow$ & $M^{-1}$ \\
$D_{P}(M)$ & $\longrightarrow$ & $1-D_{P}(M)$ \\
\hline
\end{tabular}

Table 3: Transformations that change the equations for a zero-current quasi-resonant converter into those for a zero-voltage converter.

The conversion ratio of a zero-voltage converter is given by

$$
\frac{F_{S}}{F_{0}}=\frac{1-D_{P}(M)}{G\left(R / M R_{0}\right)} .
$$

Note that the function $G$ is unchanged by the duality transformation. The small-signal model is illustrated in Fig. 11, and the parameters are given by

$$
\begin{aligned}
h_{v v} & =\left[1-D_{P}(M)\right](1-m) \\
h_{v i} & =\frac{m R\left[1-D_{P}(M)\right]}{M} \\
h_{v f} & =V_{\text {off }} \frac{\left[1-D_{P}(M)\right]}{F_{S}} \\
h_{i v} & =-\frac{m M\left[1-D_{P}(M)\right]}{R} \\
h_{i i} & =(1+m)\left[1-D_{P}(M)\right] \\
h_{i f} & =I_{\text {on }} \frac{\left[1-D_{P}(M)\right]}{F_{S}} .
\end{aligned}
$$

As before, the ratio of the subscripts gives the dimensions of each parameter.

In a full-wave zero-voltage converter, $G\left(\rho^{-1}\right)$ is nearly constant for all values $0<\rho^{-1}<1$, and $m=0$. The dc and ac behavior of zero-voltage converters is therefore the same as their zero-current counterparts. The full-wave converter behaves like a PWM converter with $1-D$ replaced by $F_{S} / F_{0}$. In half-wave zero-voltage converters, $G$ and $m$ both depend heavily on $\rho^{-1}$. These converters will exhibit dc output resistance and damping in the small-signal model.

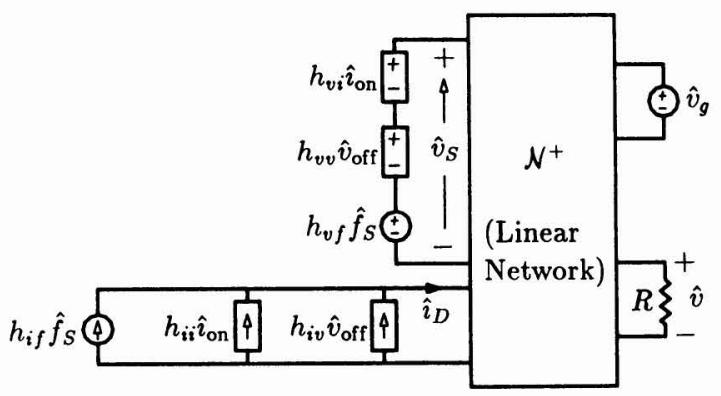

Figure 11: The small-signal model of a zero-voltage-switching converter. The switch $S$ and diode $D_{2}$ have been replaced by controlled sources according to Eqs.(36) through (41). 


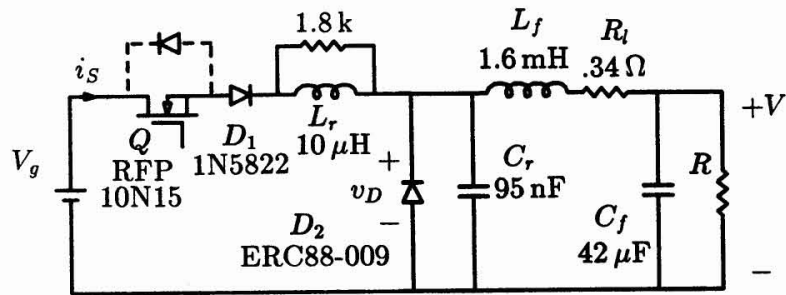

$$
\begin{array}{llrl}
L_{f}: & 105 t \# 22 \text { on TDK } & C_{f}: & 4 \times 10 \mu F, 100 \mathrm{~V}, \\
& \text { H7C4 PQ2625 core } & & \text { mylar } \\
L_{r}: & 8 t \# 20 \text { on TDK H7C1 } & C_{r}: & 2 \times .047 \mu F, 100 \mathrm{~V}, \\
& \text { RM6 core, } A_{L}=160
\end{array} \quad \begin{aligned}
& \text { polystyrene }
\end{aligned}
$$

Figure 12: Experimental resonant-switch buck converter.

\section{Experimental Measurements}

As a means of verifying the small-signal model developed in Section 6, two quasi-resonant converters were constructed and their control-to-output frequency responses measured. A VCO modulated the switching frequency while a network analyzer measured the response between the VCO input voltage and the converter output voltage. The VCO was modelled as a constant gain, a reasonable approximation for frequencies well below the switching frequency.

The first converter measured used the buck topology, as in the examples of Sections 2 and 6.3. The experimental converter is shown in Fig. 12. (The 1.8k resistor was added to damp ringing between the resonant inductance $L_{r}$ and its parasitic capacitance when the switch $S$ is open.)

Figure 13 shows the frequency response of this converter when a half-wave switch is used. The output filter alone has a $Q$ of about 5.6, but the half-wave resonant switch introduces the resistance $\left|h_{v i}\right|$ in series with the inductor $L_{f}$. At the operating point of $\rho=0.17,\left|h_{v i}\right|$ is about 0.8 times the load resistance, and the effective $Q$ of the filter is reduced to only 0.21 . The measured response clearly shows that the poles have become

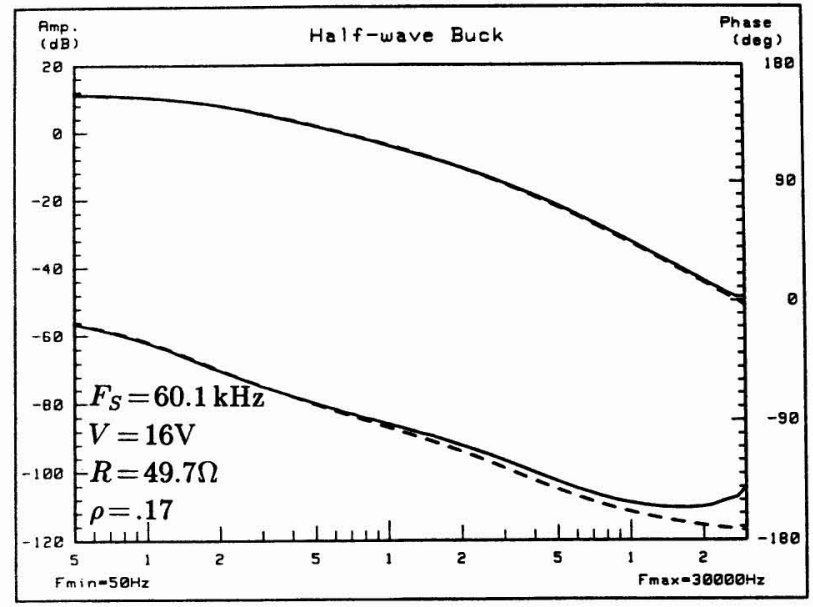

Figure 13: Measured (solid lines) and predicted (dashed lines) control-to-output frequency response of a half-wave buck converter.

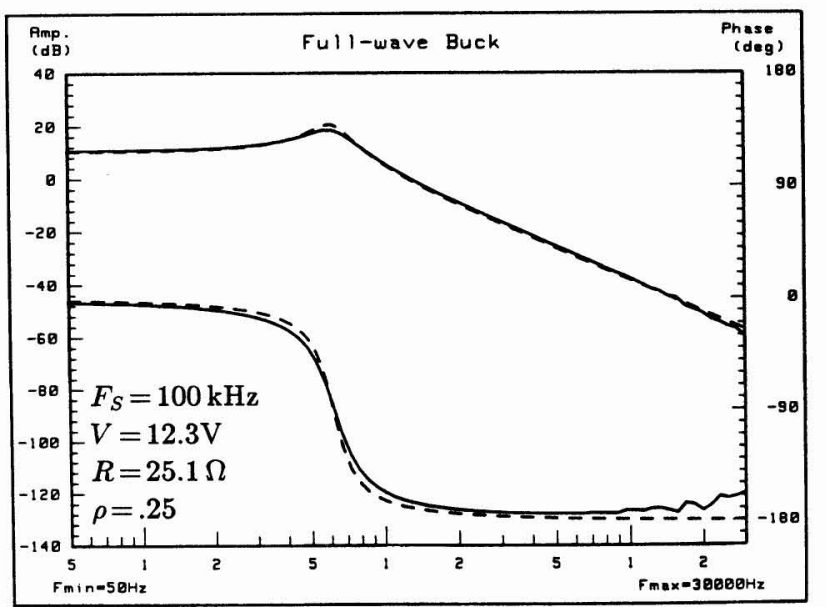

Figure 14: Measured (solid lines) and predicted (dashed lines) control-to-output response of a buck converter with a full-wave resonant switch.

real and well-separated as a result of this additional damping.

Figure 14 shows the response of the buck converter with a full-wave resonant switch (as indicated by the dashed-line position of $D 1$ in Fig. 12). The measured response exhibits the $Q$ of the output filter alone, with almost no additional damping.

Both the half- and full-wave measurements show good agreement with the small-signal model of Section 6 . The phase deviations at higher frequencies are likely a result of neglecting sampling effects in the VCO and converter.

A second converter, shown in Fig. 15, was built to verify the results of Section 7 for zero-voltage converters. The response of this boost converter with a half-wave switch appears in Fig. 16 and shows good agreement with the model of Section 7. Although the half-wave switch has introduced damping, the right-half-plane zero is still present. This will be the case for any operating point of the resonant boost converter.

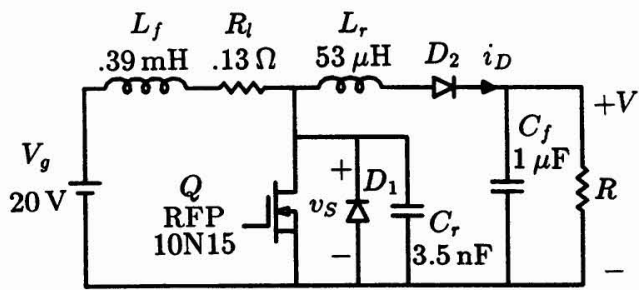

$$
\begin{array}{llrl}
L_{f}: & 67 t \# 20 \text { on } 3019 & C_{f}: & 1.0 \mu F, 100 \mathrm{~V}, \\
& \text { pot core, } 1.35 \mathrm{~mm} \text { gap } & & \text { polypropylene } \\
L_{r}: & 18 t \# 18 \text { on TDK H7C1 } & C_{r}: & 4 \times .001 \mu F, 100 \mathrm{~V}, \\
& \text { RM6 core, } A_{L}=160 & & \text { polystyrene }
\end{array}
$$

$$
D_{1}, D_{2}: \text { MUR830 }
$$

Figure 15: Experimental zero-voltage-switching boost converter. 


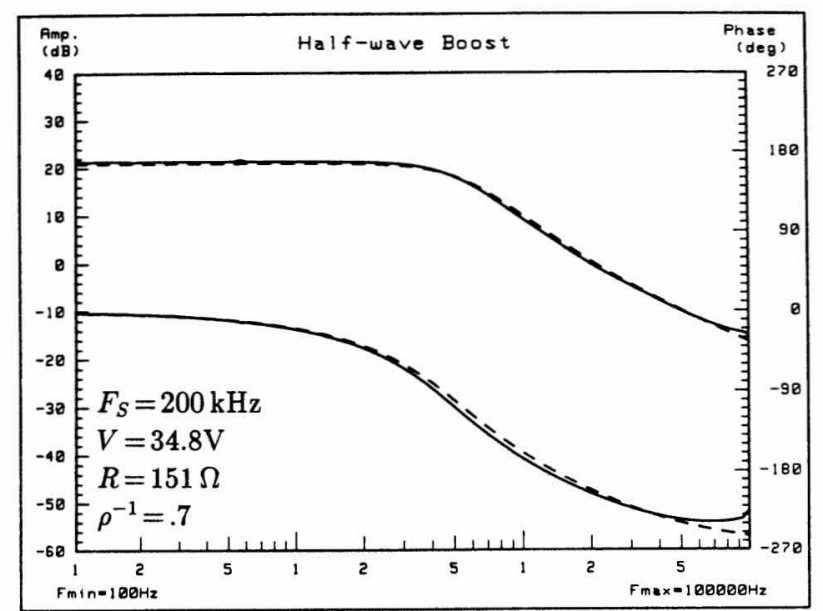

Figure 16: Measured (solid lines) and predicted (dashed lines) frequency response of the boost converter of Fig. 15.

\section{Conclusions}

Quasi-resonant converters possess a unique combination of features. With their quasi-sinusoidal waveforms, they have the desirable features of zero-current or zero-voltage switching and insensitivity to parasitics. The presence of an idle interval in the sequence of switched networks makes these converters easy to analyze, however, when compared to most other resonant converters.

Converters with resonant switches come in an astounding variety of topologies. The quasi-resonant converter is first of all based upon any one of many PWM topologies. To the basic PWM converter is added either a zero-voltage or a zero-current resonant switch. The switch can be either half-wave or fullwave, and the resonant reactances may appear in many different positions within the topology without changing the dc behavior.

By limiting consideration to a certain class of convertersthose with single switches and no coupling-the topology and behavior of the original PWM converter can be applied to the quasi-resonant converter, yielding general expressions for the $\mathrm{dc}$ conversion ratio and small-signal coefficients. The dc behavior of any converter with a resonant switch is governed by Eq.(21) or (35) for zero-current or zero-voltage converters, respectively. These equations use the known conversion ratio of the underlying PWM converters. The parameters of the small-signal dynamic model use this same ratio, giving visible evidence of the role the underlying PWM converter plays in the behavior of the resonant converter.

The general analysis performed here revealed that the qualitative behavior of a quasi-resonant converter is determined mainly by the type of resonant switch used, full- or half-wave. A converter with a full-wave switch is similar to its PWM parent in every respect. The dc conversion ratio and ac small-signal behavior (to the extent that the resonant reactances may be neglected in the small-signal model) are both obtained by substitution of the resonant control variable $f_{S} / F_{0}$ for the duty ratio $d$ which controls the PWM converter. Full-wave converters have load-independent conversion ratios and undamped dynamic responses. Half-wave converters, in contrast, demonstrate behav- ior remarkably similar to PWM converters in the discontinuous conduction mode. The conversion ratio is load-dependent, producing a lossless output resistance that fosters current-sharing among paralleled converters. The half-wave switch also introduces lossless damping in the small-signal dynamic response.

The dc analysis of quasi-resonant converters relies on the assumptions of losslessness and of small ripple in the converter's non-resonant reactances, while the small-signal analysis makes the additional approximation that modulation occurs far below the switching frequency. The loss in accuracy caused by these approximations is rewarded by the benefits of generality and simplicity in the resulting models. The results given here are offered as a means of understanding how quasi-resonant converters behave and how the many variations relate to one another and to their PWM parents.

\section{References}

[1] F. C. Schwarz, "An Improved Method of Resonant Current Pulse Modulation for Power Converters," IEEE Power Electronics Specialists Conference, 1975 Record, pp. 194204 (IEEE Publication 75CH0-965-4 AES).

[2] Dilip A. Amin, "Applying Sinewave Power Switching Techniques to the Design of High-Frequency Off-line Converters," Proc. of the Seventh National Solid-State Power Conversion Conference (Powercon 7), March 1980, pp. A1.1A1.7.

[3] K-H. Liu and F. C. Lee, "Resonant Switches-A Unified Approach to Improve Performances of Switching Converters," IEEE International Telecommunications Energy Conference, 1984 Proceedings, pp. 344-351 (IEEE Publication 84-CH-2073-5).

[4] K-H. Liu, R. Oruganti, and F. C. Lee, "Resonant Switches-Topologies and Characteristics," IEEE Power Electronic Specialists Conference, 1985 Record, pp. 106116 (IEEE Publication 85CH2117-0).

[5] K-H. Liu and F. C. Lee, "Zero-Voltage Switching Technique in Dc/Dc Converters," IEEE Power Electronics Specialists Conference, 1986 Record, pp. 58-70 (IEEE Publication $86 \mathrm{CH} 2310-1$ ).

[6] T. Zeng, D. Y. Chen, and F. C. Lee, "Variations of QuasiResonant Dc-Dc Converter Topologies," IEEE Power Electronics Specialists Conference, 1986 Record, pp. 381-392 (IEEE Publication 86CH2310-1).

[7] S. D. Freeland, "Appendix to 'A Unified Analysis of Converters with Resonant Switches," Technical Note \#195, Power Electronics Group, California Institute of Technology, March 28, 1987.

[8] R. D. Middlebrook and Slobodan Ćuk, "A General Unified Approach to Modelling Switching Converter Power Stages," IEEE Power Electronics Specialists Conference, 1976 Record, pp. 18-34 (IEEE Publication 76CH1084-3 AES); also International J. of Electronics, vol. 42, no. 6, pp. 521-550, June 1977.

[9] S. Ćuk and R. D. Middlebrook, "A General Unified Approach to Modelling Switching Dc-to-Dc Converters in 
Discontinuous Conduction Mode," IEEE Power Electronics Specialists Conference, 1977 Record, pp. 36-57 (IEEE Publication 77CH-1213-8 AES).

[10] R. P. E. Tymerski, "Generation, Classification and Analysis of Switched-Mode Dc-to-Dc Converters by the Use of Converter Cells," IEEE International Telecommunications Energy Conference, 1986 Proceedings, pp. 181-195 (IEEE Publication 86CH2328-3).

[11] Y. S. Lee, "A Systematic and Unified Approach to Modelling Switches in Switch-Mode Power Supplies," IEEE Trans. on Industrial Electronics, vol. IE-32, no. 4, November 1985, pp. 445-448.

[12] R. D. Middlebrook, "Topics in Multiple-Loop Regulators and Current-Mode Programming," IEEE Power Electronics Specialists Conference, 1985 Record, pp. 716-732 (IEEE Publication 85CH-2117-0).

[13] W. M. Polivka, P. R. K. Chetty, and R. D. Middlebrook, "State-Space Averaging Modelling of Converters with Parasitics and Storage-Time Modulation," IEEE Power Electronics Specialists Conference, 1980 Record, pp. 119-143 (IEEE Publication 80CH-1529-7). 